\title{
Design and tests of high sensitivity NTD Ge thermometers for the Planck-High Frequency Instrument
}

\author{
M. Piat ${ }^{1}$, J.P. Torre ${ }^{2}$, J.M. Lamarre ${ }^{1}$, J.W. Beeman ${ }^{3}$, R.S. Bhatia ${ }^{4}$, \\ B. Leriche ${ }^{1}$, J.P. Crussaire ${ }^{1}$, F. Langlet ${ }^{1}$ \\ 1. Institut d'Astrophysique Spatiale, Bât. 121, Université Paris XI, F-91405 Orsay, France \\ 2. Service d'Aéronomie du CNRS, BP 3, F-91371 Verrières Le Buisson, France \\ 3. Lawrence Berkeley Laboratory, University of California, Berkeley, CA 94720, USA \\ 4. California Institute of Technology, Mail Code 59-33,Pasadena, CA 91125, USA
}

\begin{abstract}
The High Frequency Instrument of Planck needs high sensitivity semi-conductors at low temperature to monitor the temperature of the bolometer plate. We have modeled such thermometers by using a semi-analytical approach of Anderson insulators, taking into account both the electrical field and the electron/phonon decoupling effects. The optimized design uses convenient NTD Ge material and has larger dimension than the initial design. The first measurements of these optimized thermometers showed a significant thermal de-coupling effect due to Kapitza resistance with its mechanical support. Nevertheless, a sensitivity of about $8 \mathrm{nK} . \mathrm{Hz}^{-0.5}$, not far from the predicted one, was obtained. The noise spectrum of the thermometer was flat down to $1 \mathrm{~Hz}$, dominated at lower frequency by the thermal fluctuations.
\end{abstract}

\section{INTRODUCTION}

The ESA satellite Planck is dedicated to survey the sky and especially the Cosmological Microwave Background anisotropies at sub-millimeter and millimeter wavelengths. To achieve this goal, the High Frequency Instrument (HFI) will use 48 bolometers cooled to $100 \mathrm{mK}$ by a space qualified ${ }^{3} \mathrm{He} /{ }^{4} \mathrm{He}$ dilution cooler. More details on Planck-HFI can be found in this proceeding [1].

Due to the scanning strategy, the detection chain has to be stable from $16 \mathrm{mHz}$ to $100 \mathrm{~Hz}[1,2]$, which defines the useful frequency range. In the space-qualified version of the dilution refrigerator, random sequences of pure ${ }^{3} \mathrm{He}$ bubbles in the ${ }^{3} \mathrm{He} /{ }^{4} \mathrm{He}$ diluted phase produce low frequency fluctuations of the $100 \mathrm{mK}$ stage temperature [3] that can be detected by bolometers as a signal. It has been shown [4] that a $100 \mathrm{mK}$ bolometer plate temperature stability of $20 \mathrm{nK}^{-\mathrm{Hz}^{-0.5}}$ will degrade the overall HFI sensitivity by less than $5 \%$, which defines the HFI $100 \mathrm{mK}$ temperature stability requirement.

This requirement will be reached using a combination of a passive thermal filter [2, 4] and an active regulation stage. Therefore, Planck-HFI needs high sensitivity thermometers both for temperature fluctuations monitoring and temperature regulation. In order to have a reasonable safety margin, the goal was set to reach a sensitivity level of $10 \mathrm{nK} . \mathrm{Hz}^{-0.5}$ between $16 \mathrm{mHz}$ and $100 \mathrm{~Hz}$.

CP605, Low Temperature Detectors, edited by F. S. Porter et al. (c) 2002 American Institute of Physics 0-7354-0049-0/02/\$19.00 
In this paper, we present the design and the tests of high sensitivity NTD Ge thermometers at low temperature. First experimental results exhibit thermal decoupling that does not strongly affect the measured sensitivity.

\section{DESIGN OF HIGH SENSITIVITY NTD GE THERMOMETERS}

We define the responsivity as Resp $=\mathrm{dV} / \mathrm{dT}_{\mathrm{ph}}$ where $\mathrm{V}$ is the thermometer voltage bias and $T_{p h}$ the phonon temperature to measure. It is related with the sensitivity $S$ (expressed in $\mathrm{K} . \mathrm{Hz}^{-0.5}$ ) by $\mathrm{S} \times \mathrm{Resp}=\mathrm{ASDV}$, where ASDV is the Amplitude Spectral Density of V (expressed in V.Hz ${ }^{-0.5}$ ). Responsivity can be used as a guideline for optimisation since we generally find that it varies more rapidly than sensitivity with all free parameters.

In the case of a perfect thermometer with a resistance depending only on temperature, the responsivity increases simply by increasing the bias current (BC) I. However, real thermometers at low temperature exhibit two non-linear effects that degrade somewhat their sensitivity.

The electrical field effect for variable range hopping in a disordered semi-conductor can be expressed as follows, in the limit of small and uniform electrical field E [5]:

$$
\mathrm{R}\left(\mathrm{V}, \mathrm{T}_{\mathrm{e}}\right)=\mathrm{R}_{0} \exp \left(\frac{\mathrm{T}_{0}}{\mathrm{~T}_{\mathrm{e}}}\right)^{\mathrm{m}} \exp \left(-\frac{\mathrm{eVL} \mathrm{L}_{\mathrm{h}}}{\mathrm{k}_{\mathrm{B}} \mathrm{T}_{\mathrm{e}} \mathrm{L}}\right) \text { for } \mathrm{E}=\frac{\mathrm{V}}{\mathrm{L}}<<\frac{\mathrm{mk}_{\mathrm{B}} \mathrm{T}_{\mathrm{e}}}{\mathrm{eL}_{\mathrm{h}}}
$$

where $R_{0}, T_{0}$ and $m$ are very low bias power parameters, $T_{e}$ is the electronic temperature, $e$ is the electron charge, $L_{h}$ is a characteristic length for the hopping process, $\mathrm{L}$ is the inter-electrodes length and $\mathrm{k}_{\mathrm{B}}$ is the Boltzmann constant. The characteristic hopping length is expected to vary as $\mathrm{L}_{\mathrm{h}}\left(\mathrm{T}_{\mathrm{e}}\right)=\mathrm{L}_{\mathrm{h} 0} \times \mathrm{T}_{\mathrm{e}}^{-\mathrm{m}}$ where $\mathrm{L}_{\mathrm{h} 0}=\mathrm{L}_{\mathrm{h}}\left(\mathrm{T}_{\mathrm{e}}=1 \mathrm{~K}\right)[5]$.

The electron/phonon decoupling effect can be modeled by the following expression [6]:

$$
\mathrm{P}=\mathrm{G}_{\mathrm{e}-\mathrm{ph}} \operatorname{Vol}\left(\mathrm{T}_{\mathrm{e}}^{\mathrm{n}}-\mathrm{T}_{\mathrm{ph}}^{\mathrm{n}}\right)
$$

where $\mathrm{P}=\mathrm{VI}$ is the bias power, $\mathrm{G}_{\mathrm{e}-\mathrm{ph}}$ is the coupling factor, $\mathrm{Vol}$ is the volume of the device and $\mathrm{n}$ is a constant found experimentally close to 6 for NTD Ge [6].

At any $B C$, the bias voltage across the sample must verify $V=R\left(V, T_{e}\right) \times I$ which can be solved numerically. When phonon temperature of the sample could not be well matched to the heat-sink temperature, a first order correction was introduced with an estimation of the equivalent thermal conductance to the base plate.

This model was implemented within the IDL environment. It was validated on NTD $\mathrm{Ge}$ thermometer and on $\mathrm{NbSi}$ thin film [7]. It allowed us to evaluate the non-linearity parameters $\left(\mathrm{G}_{\mathrm{e}-\mathrm{ph}}\right.$ and $\left.\mathrm{L}_{\mathrm{h} 0}\right)$ of Haller-Beeman Associates (H-B) NTD Ge materials and to calculate theoretical sensitivities with different geometry, doping level and BC.

As shown on figure 1, this optimization process leads to the choice of two NTD Ge thermometers with size larger than usual: $\mathrm{H}-\mathrm{B}$ type $\mathrm{H}$ with $400 \mu \mathrm{m}$ inter-electrodes length and a volume of $400 \times 250 \times 250 \mu \mathrm{m}^{3}$ and $\mathrm{H}-\mathrm{B}$ type $\mathrm{G}$ with $400 \mu \mathrm{m}$ interelectrodes length and a volume of $400 \times 1000 \times 1000 \mu \mathrm{m}^{3}$. 

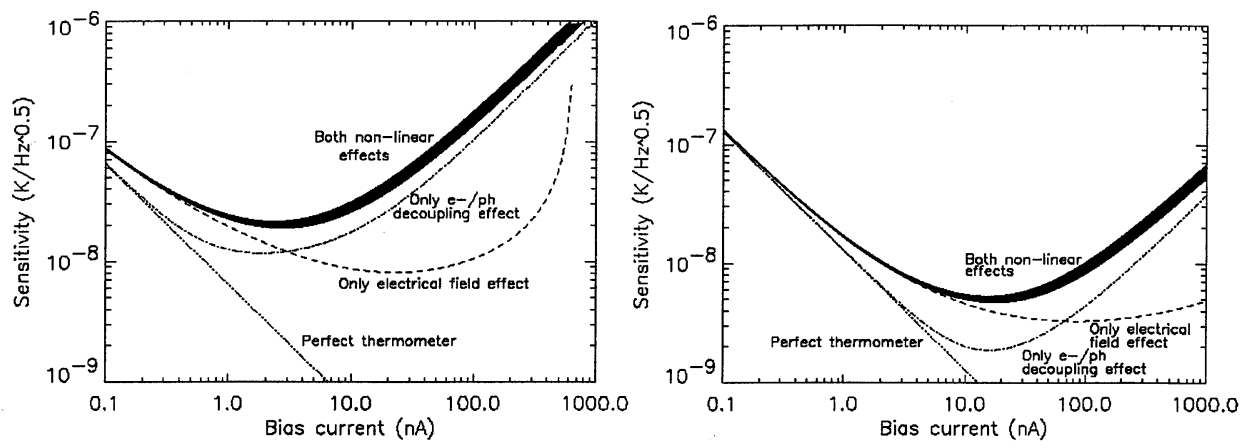

FIGURE 1. Simulated sensitivities of $H-B$ NTD Ge G materials at $100 \mathrm{mK}$ as a function of the BC, taking into account the readout noise $\left(\mathrm{e}_{\mathrm{n}}=5 \mathrm{nv} . \mathrm{Hz}^{-0.5}, \mathrm{i}_{\mathrm{n}}=0.5 \mathrm{fA} . \mathrm{Hz}^{-0.5}\right)$ and the Johnson noise. On the left graph, a cubic geometry of $(250 \mu \mathrm{m})^{3}$ is assumed while the right one is for a $400 \times 1000^{2} \mu \mathrm{m}^{3}$ (see text). The bold curves contain all cases at $\pm 1 \sigma$ on $\mathrm{G}_{\mathrm{e} \text {-ph }}$ and $\mathrm{L}_{\mathrm{h} 0}$. Parameters are: $\mathrm{R}_{0}=2.026 \Omega$ (for $250 \mu \mathrm{m}^{3}$ geometry); $\mathrm{T}_{0}=30.815 \mathrm{~K} ; \mathrm{m}=0.5 ; \mathrm{G}_{\mathrm{e}-\mathrm{ph}}=(10.9 \pm 1.5) \mathrm{W} / \mathrm{K}^{6} / \mathrm{cm}^{3} ; \mathrm{n}=6 ; \mathrm{L}_{\mathrm{h} 0}=(24.5 \pm 1.3) \mathrm{nm}$.

\section{FIRST TEST OF LARGE NTD Ge THERMOMETERS}

The NTD Ge crystals have been mounted as shown on figure 2. The copper setting is a screw in order to ease its positioning on the cold plate. Two sets of increased sizes H-B type G and H-B type H NTD Ge thermometers have been tested on the Symbol cryostat: one is glued with silver epoxy and the other with gold epoxy.

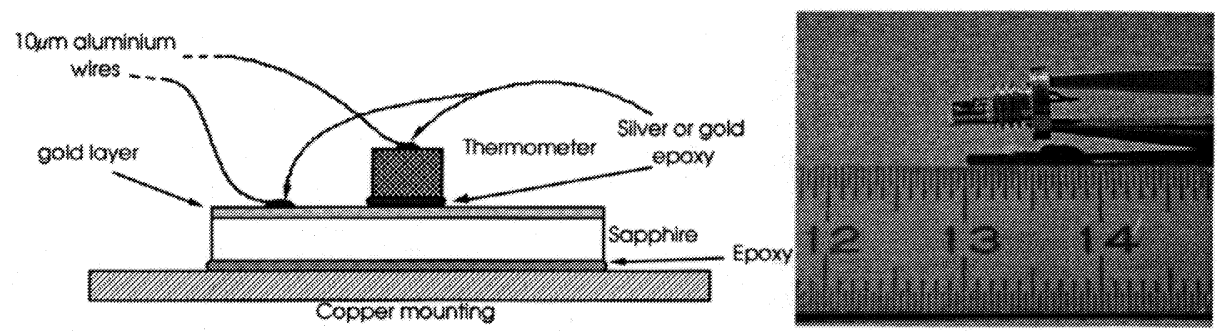

FIGURE 2. Setting of the tested H-B thermometers.

The thermal architecture of Symbol is a two-stage passive low-pass filter, the first one supporting the regulation system. It allows us to obtain a temperature stability better than $20 \mathrm{nK} . \mathrm{Hz}^{-0.5}$ down to about $0.1 \mathrm{~Hz}[2]$.

The readout electronics are based on a square bias electrical modulation obtained through a capacitive load impedance [8,9]. This system has an input noise level of less than $5 \mathrm{nV} . \mathrm{Hz}^{-0.5}$ in the useful frequency band and has been calibrated in order to reduce the uncertainty on the bias current to about $1 \%$ [7].

The first characterization of these thermometers has shown a non-negligible thermal conductance to the base plate mainly due to Kapitza resistance between the sample and the sapphire board. The value of this conductance estimated from our simulations is consistent with a $\mathrm{T}^{4}$ law and is about $7.10^{-6} \mathrm{~W} / \mathrm{K} / \mathrm{cm}^{2}$ for gold epoxy and $3.10^{-6}$ $\mathrm{W} / \mathrm{K} / \mathrm{cm}^{2}$ for silver epoxy at $100 \mathrm{mK}$. This introduces an excess noise term of about 
$4 \mathrm{nK} . \mathrm{Hz}^{-0.5}$. This thermal decoupling may be increased by stress problems that have been encountered on some device. Figure 3 represents a temperature noise spectrum obtained with this set-up. The thermal fluctuations of the cryogenic system dominate at frequency lower than $1 \mathrm{~Hz}$. The ultimate sensitivity is about $8 \mathrm{nK} \cdot \mathrm{Hz}^{-0.5}$, which is consistent with the model.

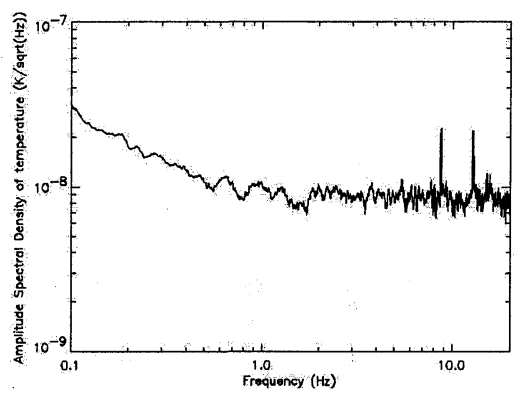

FIGURE 3. Amplitude spectral density of the temperature measured at $102 \mathrm{mK}$ with $\mathrm{H}-\mathrm{B}$ Ge NTD G thermometer with a volume of $495 \times 1050 \times 1050 \mu \mathrm{m}^{3}(\mathrm{R}=3 \mathrm{M} \Omega, \mathrm{I}=10 \mathrm{nA})$. This device was placed on the bolometer plate of the Symbol thermal architecture V2.1. The increase at low frequency is attributed to thermal fluctuations of the cryogenic system.

\section{CONCLUSION}

A NTD Ge thermometer suitable for the Planck-HFI instrument has been designed thanks to our modeling and tested on the Symbol cryostat. Although a thermal decoupling was present due to Kapitza resistance with the mounting, a sensitivity of about $8 \mathrm{nK} . \mathrm{Hz}^{-0.5}$ has been obtained and the HFI requirement is met at least down to about $0.4 \mathrm{~Hz}$.

\section{ACKNOWLEDGMENTS}

We thank P. Garoche at Laboratoire de Physique des Solides in Orsay for fruitful discussions. The Symbol experiment is funded by CNES and supported by IAS.

\section{REFERENCES}

1. Lamarre J.M., this proceeding.

2. Piat M. et al., Proc. of LTD-8, NIMA, 444, 419-422 (2000).

3. Camus P., Analyse du fonctionnement d'un cryostat à dilution en cycle ouvert pour les applications spatiales, CRTBT training period report, 1998.

4. Piat M., Contributions à la définition des besoins scientifiques et des solutions instrumentales du projet Planck-HFI, PhD thesis, 2000.

5. Kenny T.W. et al., Phys. Rev. B 39, 8476 (1987).

6. Wang N. at al., Phy. Rev. B 41, 6, 3761-3768 (1990).

7. Piat M. et al., JLTP, submitted.

8. Gaertner S. et al., Astron. Astrophys., Suppl. Ser., 126, 151-160 (1997).

9. Piat M. et al., Proc. LTD-7, 27 July - 2 August 1997, Munich, Germany, pub. by MPI Physik, ISBN 3-00-002266-X. 